\title{
II. MITTEILUNGEN
}

\section{Professor Viktor Dieterich 85 Jahre alt}

Der em. o. ö. Professor für Forstpolitik und Forstliche Betriebswirtschaftslehre an der Universität München, Dr. Dr. h. c. V. DieTErich, feiert am 26. August seinen 85. Geburtstag.

Eine Würdigung der umfangreichen Lebensarbeit des Jubilars ist bereits im Jahrgang 1949 unserer Zeitschrift erschienen. Dem damals veröffentlichten Schriftenverzeichnis wäre eine Reihe weiterer Arbeiten hinzuzufügen, da der Jubilar nach wie vor als Gutachter und wissenschaftlicher Schriftsteller tätig ist. In diesem Zusammenhang kann auf eine Reihe von Beiträgen aus seiner Feder in den letzten Jahrgängen des Forstwissenschaftlichen Centralblattes verwiesen werden.

Dem bedeutenden Gelehrten und langjährigen rührigen Mitarbeiter unserer Zeitschrift dürfen aus Anlaß dieses Festtages die herzlichsten Glückwünsche ausgesprochen werden.

\section{Forstliche Hochschulwoche 1964}

Die diesjährige Hochschulwoche findet in Freiburg in der Zeit vom 26. bis 30. 10. 1964 statt. Für den 26. bis 29. 10. 1964 sind Fachvorträge und für den 30. 10. 1964 Exkursionen vorgesehen.

\section{Verleihung des Wilhelm-Leopold-Pfeil-Preises}

Die gemeinnützige Stiftung F.V.S. zu Hamburg hat einen Wilhelm-Leopold-Pfeil-Preis neu zur Verfügung gestellt. Der mit $10000,-$ DM dotierte Preis soll alljährlich Persönlichkeiten aus Wissenschaft und Praxis verliehen werden, die sich um eine, auch auf die Zukunft gesehen, beispielhafte Waldwirtschaft in Europa besonders verdient gemacht haben. Dabei sollen auch die Erfordernisse berücksichtigt werden, die von der Allgemeinheit in sozialer, hygienischer und ästhetischer Hinsicht immer nachdrücklicher vertreten werden.

Der Preis trägt den Namen des ersten Direktors der Forstakademie in Eberswalde, Wilhelm-Leopold-Pfeil, der sich um die wissenschaftliche Forstwirtschaft in ihren Anfängen in der ersten Hälfte des 19. Jahrhunderts sehr verdient gemacht hat.

Das Preis-Kuratorium unter dem Vorsitz von Professor Dr. Julrus Speer hat beschlossen, den Preis für das Jahr 1963 Professor Dr. Dr. h. c. V. Dieterich, Stuttgart, dem vielleicht letzten Universalvertreter der Forstwissenschaft, und den Preis für 1964 Professor Dr. Gustay Krauss, München, als hervorragendem Vertreter der Standortskunde zuzuerkennen. Beide Persönlichkeiten haben sich große Verdienste um Forstwissenschaft und -wirtschaft erworben.

Außerdem werden 4 deutsche und 4 ausländische europäische Forststudenten oder in der weiteren Ausbildung befindliche junge Forstleute mit Stipendien von insgesamt $10000,-\mathrm{DM}$ ausgezeichnet, um ihnen die Möglichkeit zu geben, ihre Kenntnisse in anderen europäisctien Ländern $\mathrm{zu}$ erweitern.

Die Preise werden durch die Albert-Ludwigs-Universität in Freiburg bei der Eröffnung der forstwirtsduaftlichen Hochschulwoche Ende Oktober dieses Jahres verliehen. 